todo en él es genio, invención, innovación y talento creativo. Por consiguiente, la verdadera base para la creación poética de Benavente es la lengua misma, la cual él maneja con una confrontación directa en tres polaridades indisolubles: lengua diaria/lengua poética, Leandro/ Crispín, y norma/ruptura, repitiendo en la obra la misma técnica que ha empleado en el título, o sea una dinámica transformación de la lengua diaria en lengua poética. El propósito de este trabajo ha sido demostrar los medios que Benavente emplea para efectuar esta transformación propuesta por él mismo en su prólogo a Los intereses creados.

Robert J. Young

Northern Illinois University.

\title{
LA DISPERSIÓN DE LA PALABRA: APROXIMACIÓN LIGÚÍSTICA A POEMAS "VALLEJO"
}

La lingüística, y particularmente las extensiones de la lingüística que se ocupan de explicar el lenguaje poético, han resucitado la importancia que la retórica otorgó al discurso y que el romanticismo eclipsó bajo su teoría de la expresión. A partir de la perspectiva lingüística comienza a crearse un nuevo espacio teórico, cuyo postulado básico no es ni la mimesis (imitación de las acciones de los hombres, representación de la naturaleza o de la sociedad, según las variantes históricas) ni la expresión (o "sensibilización de la idea", que es la fórmula con que Hegel resume las teorías románticas), sino la función (poética) del lenguaje. Mi propósito es indagar, a través de la lectura de algunos poemas "Vallejo"1, ciertos aspectos de la tentativa lingüística de fundar una poética (tomada aquí como equivalente de 'teoría del discurso poético') y, dentro del mismo espacio teórico abierto por ella, señalar sus límites.

La tentativa no es nueva, por supuesto. Sus comienzos sistemáticos se encuentran en el formalismo ruso: "El establecimiento de dos nociones -lengua y habla - y el análisis de sus interrelaciones (escuela de Ginebra) fueron en extremo fecundos para la lingüística. Aplicar estas categorías (la norma existente y los enunciados individuales) a la literatura

1 Las comillas de "Vallejo" indican que, al escribir el nombre del poeta, no tomo en cuenta posibles conexiones entre el texto y el autor. Tampoco supongo "intenciones". Las consideraciones sobre el autor y sus intenciones, si bien importantes, escapan a una poética (como teoría) tal como aquí la concibo. Pienso en la observación de Paul Zumthor, Essai de poétique médiévale, Paris, 1972, p. 11: "Le texte est le produit d'une opération d'encodage, pratiquée à partir de l'intégrité structurelle et culturelle d'un état de langue. Un élément intentionnel y a été introduit, qui a pour fonction d'orienter le décodage: mais rien ne résiste plus mal à la durée que cet effet. Dans le meilleur des cas, les décodages successifs actualisent les potentialités toujours nouvelles du texte" (el subrayado es mío). -Para Los Heraldos Negros me sirvo de la ed. de Buenos Aires (Losada), 1969. - Mi agradecimiento a Noé Jitrik por su lectura del manuscrito de este artículo y por sus sugerencias críticas. 
y estudiar sus interrelaciones, es un problema que debe estudiarse a fondo" 2 . Este planteo, recurrente en los trabajos de los formalistas rusos, reaparece en 1958 en Roman Jakobson. A la pregunta “¿Qué es lo que hace de un mensaje verbal una obra de arte?", Jakobson da su célebre respuesta: "El lenguaje poético desplaza el principio de equivalencia del eje de la selección al eje de la combinación"3 - hipótesis que es extensión de otra mediante la cual explica el mismo Jakobson el funcionamiento de la "lengua comunicacional"4: "Hablar implica seleccionar ciertas entidades lingüísticas y combinarlas en unidades lingüísticas de mayor complejidad". En esta operación "el receptor percibe que el enunciado dado (mensaje) es una combinación de partes constituyentes (frases, palabras, fonemas, etc.) seleccionadas en el repertorio de todas las partes constituyentes posibles (código)". En esta doble operación hay que distinguir dos planos: "los constituyentes de un contexto están en situación de contigüidad, mientras que en un grupo de sustitución los signos están ligados entre sí por diferentes grados de similaridad". De ello se sigue que la metáfora opera en el eje de la selección (por similaridad) y la metonimia en el eje de la combinación (por contigüidad). Cuando estas operaciones se ejercen sobre un enunciado lingüístico en cualquiera de sus niveles -morfológico, sintáctico, léxico o fraseológico-, tal operación asume la forma del siguiente diagrama:

$$
A
$$

Selección

equivalencia

similaridad

metáfora
C

Niveles

enunciado
lingüístico
$B$

\section{Combinación}

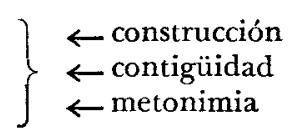

Con base en tal esquema, un enunciado de la lengua comunicacional se presenta como un enunciado del tipo

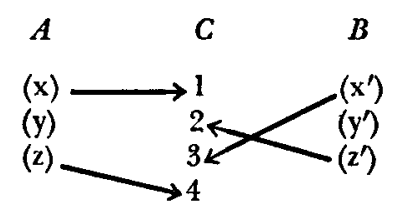

en el cual no existe el encabalgamiento, y cada una de las variables $\left(x, y, z, x^{\prime}, y^{\prime}, z^{\prime}\right.$ ) de los dos ejes (selección y combinación) operan sobre diferentes planos del enunciado. Así,

$2 \mathrm{~J}$. Tynianov et $R$. Jakobson, "Les problèmes des études littéraires et linguistiques", en Théorie de la littérature, ed. T. Todorov, Paris, 1965, p. 140. Sobre el formalismo ruso véase el importante libro de Victor ERLICH, Russian formalism, The Hague, 1955, en especial la segunda parte ("The theory").

3 R. JAKoBson, "Linguistics and poetics", en Style in language, Cambridge, Mass., 1960, p. 358. (Hay traducción francesa en R. JAKOBson, Essais de linguistique générale, Paris, 1963).

4 R. Jakobson, "Deux aspects du langage et deux types d'aphasie”, en sus Essais de linguistique générale, op. cit., pp. 45-49. 


$$
((\mathrm{x}) 1) ;\left(\left(\mathrm{x}^{\prime}\right) 3\right) ;((\mathrm{z}) 4) ;\left(\left(\mathrm{z}^{\prime}\right) 2\right) \text {. }
$$

Por el contrario, en el lenguaje poético, al proyectarse el principio de equivalencia del eje de la selección al eje de la combinación y la similaridad sobre la contigüidad, el enunciado es del tipo

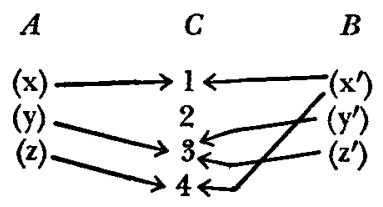

en el cual se transforma la secuencia (3) en una doble convergencia de variables:

$$
\left(\left(x, x^{\prime}\right) 1\right) ;\left(\left(y, z^{\prime}\right) 3\right) ;\left(\left(y, y^{\prime}\right) 3\right) ;\left(\left(z, x^{\prime}\right) 4\right)
$$

o bien:

$$
\left(\left(y, y^{\prime}, z^{\prime}\right) 3\right)
$$

Convergencia en la proyección que, como dice Jakobson, hace a toda metáfora ligeramente metonímica y da a toda metonimia un tinte metafórico ${ }^{5}$.

Si hemos insistido en este aspecto ya clásico de la poética lingüística, ha sido porque lo que en Jakobson es una hipótesis (cf. infra, p. 410), en S. Levin ${ }^{6}$ se desarrolla como modelo. Sobre él nos detendremos.

Para hacer aparecer lás convergencias señaladas en (4), (5) y (6), Levin comienza por introducir un concepto fundamental: el concepto de acoplamiento ("coupling"). La necesidad básica para la elaboración de todo modelo es la definición de conceptos que permitan derivar otros a partir de ellos. Dicho de otro modo: hacer un modelo es construir un discurso, definiendo y relacionando conceptos, a nivel de la lógica 7 . Así, la definición de acoplamiento se hace a partir de una reformulación de las nociones de sintagma y paradigma. Criticando a ciertos lingüistas por su tendencia a privilegiar al primero en perjuicio del segundo, Levin insiste en su interrelación: el paradigma se funda en el sintagma y el sintagma en el paradigma. Su reivindicación del paradigma importa en la medida en que éste es fundamental en el lenguaje poético: "consideramos el poema no sólo como una sucesión de sintagmas, sino mejor/ también [rather/also] como un sistema de paradigmas" (p. 19).

5 Recordemos, como información adicional, el desarrollo de la noción de metonimia llevado a cabo en el análisis del relato por G. Genette, "Métonymie chez Proust, ou la naissance du récit", Poétique, 1969, núm. 2, 156-173.

6 Samuel Levin, Linguistic structures in poetry, The Hague, 1963.

7 Sobre la noción de modelo remito a H. Freudenthal, The concept and the role of the model in mathematics and natural and social sciences, Dordrecht, 1961, y a la Parte IX ("Methodology and philosophy of linguistics") del Symposium on Models in Linguistics, ed. por TARski, NAGeL \& SUPPES, Logic, methodology and philosophy of science, Stanford Univ. Press, 1962, pp. 528-583. 
La noción de acoplamiento se funda, pues, en la importancia del paradigma y en su interrelación con el sintagma. Antes de volver sobre ella, introduciré un ejemplo sobre el cual haré unas observaciones (guiado por el modelo de Levin), que me servirán de apoyo para consideraciones posteriores.

\section{Los Heraldos Negros}

1 Hay golpes en la vida, tan fuertes... Yo no sé! Golpes como del odio de Dios; como si ante ellos, la resaca de todo lo sufrido se empozara en el alma... Yo no sé!

5 Son pocos, pero son... Abren zanjas oscuras en el rostro más fiero y en el lomo más fuerte. Serán tal vez los potros de bárbaros atilas; o los heraldos negros que nos manda la Muerte.

Son las caídas hondas de los Cristos del alma,

10 de alguna fe adorable que el Destino blasfema. Esos golpes sangrientos son las crepitaciones de algún pan que en la puerta del horno se nos quema.

Y el hombre... Pobre... pobre! Vuelve los ojos, como cuando por sobre el hombro nos llama una palmada;

15 vuelve los ojos locos, y todo lo vivido se empoza, como un charco de culpa, en la mirada.

Hay galpes en la vida, tan fuertes... Yo no sé!

Haré, ante todo, estas observaciones:

(a) "todo lo sufrido" (v. 3), "todo lo vivido" (v. 15), enunciados que son sintagmas nominales (SN) del tipo: grupo sustantival (gS) + identificadores, que son género (gn) y cuantificador $(Q)$; responden, pues, a la estructura $\mathrm{SN}=\mathrm{gS}+\mathrm{Q}+\mathrm{gn}$;

(b) "en la vida" (v. 1), "en el rostro" (v. 6), "en el lomo" (v. 6), enunciados que son sintagmas circunstanciales (SC) compuestos de preposición (pr) + grupo sustantival, o sea $\mathrm{SC}=\mathrm{gS}+\mathrm{pr}$;

(c) "en el rostro más fiero y en el lomo más fuerte" (v. 6) : dos enunciados, ligados por la conjunción $y$, del tipo $\mathrm{SC}+\mathrm{Q}+\mathrm{A}$ (adjetivo), o sea: $\mathbf{E}:=(\mathrm{SC}+\mathrm{Q}+\mathrm{A}) V(\mathrm{SC}+\mathrm{Q}+\mathrm{A})$, donde $V$ es el símbolo de conjunción lógica equivalente a la copulativa y (observo también que rostro y lomo aparecen en el mismo verso como sinécdoques de 'cuerpo') ;

(d) "Serán tal vez los potros de bárbaros atilas; / o los heraldos negros que nos manda la Muerte" (vs. 7-8) : dos enunciados precedidos por el mismo verbo (serán); definiciones de golpes y, al mismo tiempo, metonimias de 'sufrir';

(e) "Son las caídas hondas de los Cristos del alma, / de alguna fe adorable que el Destino blasfema": otros dos enunciados precedidos por 
un mismo verbo (son): también definiciones de golpes y metonimias de 'sufrir';

(f) "tan fuertes" (v. 1), "más fiero" (v. 6), "más fuerte" (v. 6), "sangrientos" (v. 11), "tan fuertes" (v. 17), grupo en el cual tenemos: (fI) fuertes/fiero/fuerte/fuertes (vs. $1,6,6,17$ ), que son adjetivos precedidos por $Q$, y (f2) fuertes/sangrientos/fuertes (vs. 1, 11, 17), que operan como modificadores (M) de golpes.

Estamos ya en condiciones de retomar el concepto de acoplamiento.

En las observaciones precedentes, hay en (a) dos enunciados del tipo $\mathrm{SN}=\mathrm{gS} \mp \mathrm{Q}+\mathrm{gn}$, los cuales son equivalentes por posición. Lo mismo se puede decir de los casos (b) y (c). Los enunciados equivalentes por posición definen una clase de equivalencias: clase de equivalencia PostCIONAL, O TIPO I (Tl).

Ahora bien, las equivalencias pueden ser paralelas o comparables. En (a) y en (b) -como también en (f1) - se trata de equivalencias paralelas, en cuanto que son enunciados de construcción sintáctica similar. En (d) y en (e), por el contrario, se trata de equivalencias comparables, en cuanto que son definiciones de un mismo sustantivo (golpes) y metonimias de un mismo concepto ('sufrir'). Son también equivalencias comparables las anotadas en (f2).

Las equivalencias $\mathrm{T} 1$ se definen, pues, sobre la noción de paradigma, como "sistema de variación" que opera sobre dos enunciados en relación de posición. Dicho de otro modo: las equivalencias T1 (paralelas o comparables) aparecen, en el sistema paradigmático de variación, por su posición en el sintagma. Levin define la clase de equivalencia T1 como interior o lingüística; lo cual quiere decir que hay otro tipo de equivalencias.

En efecto, si en (a) señalé dos enunciados paralelos por posición, también vivido/sufrido pueden ser señalados como equivalentes por sinonimia y por fonías, y lo mismo ocurre con fiero/fuerte en (c) y en (fl). Este segundo tipo de equivalencias, fundado en la semántica y/o la fonía, define la clase de equivalencias de TIPo II (T2) o NATURAL (donde natural no denota otra cosa que la equivalencia T2). Levin define esta clase de equivalencias como exterior o extralingüística.

El acoplamiento aparece cuando dos enunciados (o partes de enunciados), sintácticamente similares (función relacional en el sintagma), y que por lo tanto establecen equivalencias posicionales o $\mathrm{T} 1$, convergen con equivalencias naturales o $\mathbf{T} 2$ (función co-relacional en el paradigma). Si (y sólo si) hay doble convergencia, se puede hablar de acoplamiento, estructura que es fundamental en poesía. Escribiendo $\mathrm{Sg}$. por sintagma y Pg por paradigma, esta convergencia puede representarse así:

8 Hablo aquí de sinonimia referencial, y no en el sentido que el término adquiere en los análisis de K. HEGER, "L'analyse sémantique du signe linguistique", Langue Française, 4 (1969), 44-66. Las equivalencias semánticas pueden establecerse también por antonimia (lo mojado vs. lo seco, el agua vs. el fuego, tal como ocurre en el último poema de Trilce). Esto debe entenderse en el impreciso campo en que S. Levin define la semántica: como "thought mass" o relación intuitiva, en un grupo lingüistico, entre un par o un conjunto de palabras. 


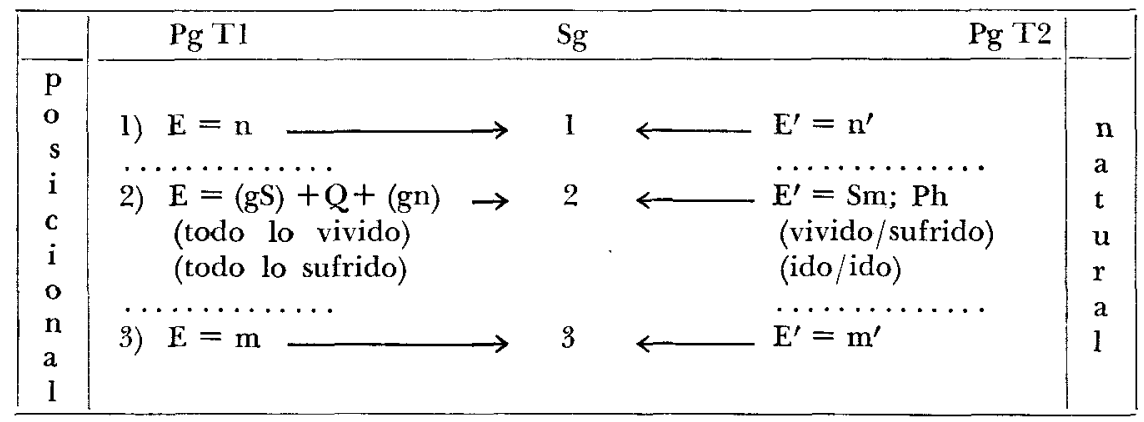

donde se escribe $\mathrm{Sm}$ por semántica y $\mathrm{Ph}$ por fonía, y donde $\mathrm{n}, \mathrm{n}^{\prime}, \mathrm{m}, \mathrm{m}^{\prime}$ representan otras posibles convergencias a establecer en la lectura del poema.

Así, pues, un poema es una serie de convergencias ( $T 1, T 2)$ en las cuales, como dice Levin, "las formas que se encuentran en acoplamientos poéticos son, al mismo tiempo, miembros de dos paradigmas distintos: uno T1, y otro T2". De ese modo reaparece la hipótesis de Jakobson, desarrollada a través de un modelo descrito a partir de un concepto de base (acoplamiento) y dos conceptos secundarios (Pg T1 y Pg T2) con dos predicados cada uno (paralelo y compárable para $\mathrm{T} 1$; semántico y fónico para T2). Por lo tanto, el acoplamiento (Cp) es una frase del tipo

$$
\mathrm{Cp}=(\mathrm{Pg} \mathrm{T} 1)(\mathrm{p} . V \mathrm{c} .) \longleftrightarrow(\mathrm{Pg} \mathrm{T} 2)(\mathrm{Sm} V \mathrm{Ph}),
$$

donde $(\longleftrightarrow)$ indica la convergencia.

Después de describir el modelo, Levin se propone una tarea sobre cuya pertinencia podría ponerse un signo de interrogación: explicar la unidad del poema. En primer lugar, hay que señalar que proponerse dar cuenta de "la unidad de un poema" (o de una obra) no es cosa inocente: se mantiene intacto un concepto establecido por el neoclasicismo y adoptado por el romanticismo ${ }^{9}$. En segundo lugar, hay que señalar que la explicación de la unidad del poema viene, por un lado, a dar sentido a la construcción del modelo, mientras que, por otro lado, aparece como un "agregado" a la construcción de un modelo que partía del a priori de que el lenguaje poético se define en relación al lenguaje comunicacional, y para cuyo funcionamiento (el del lenguaje poético) se ha construido el modelo. Todo ello obliga a Levin a "saltar", para explicar la unidad del poema, del modelo a la introducción de nuevos conceptos (extraños al modelo) y que caen del cielo: para explicar el Soneto XXX de Shakespeare afirma, por ejemplo, que "la construcción de la totalidad del poema consiste en dos frases condicionales y una conclusión" (p. 52).

Me detengo, por el momento, en este "salto" y en la introducción de conceptos exteriores al modelo. De este modo, el poema de mi ejem-

9 Véase, sobre el particular, el interesante libro de M. H. Aвrams, The mirror and the lamp: romantic theory and critical tradition, New York, 1953. 
plo puede explicarse en su unidad (siguiendo el juego de Levin) como dos proposiciones de existencia y una conclusión. Así:
A. Proposición de existencia: Hay... (v. 1).
B. Proposiciones que definen a A: son, serán (vs. 2, 5, 7, 8, 9, 10, 11).
C. Proposiciones que explican los efectos de A: abren... (vs. 5, 6) .
D. Proposición de existencia implícita: (Hay) el hombre... (v. 13).
E. Proposiciones de los efectos de A (B y C) en D (vs. 14, 15, 16).
$A^{\prime}$. Repetición de la proposición de existencia (v. 17).

De esta manera, el poema puede ser escrito como una frase del tipo

$$
\text { Si A (que B y C), entonces D (que E), }
$$

lo cual hace innecesaria la fórmula (8). Por otra parte, si como punto de llegada del modelo se establece la explicación de la unidad del poema, es inevitable la introducción de nuevos conceptos. $Y$ ello es indudable así, puesto que, aun agregando otros acoplamientos a los ejemplos anteriores -observaciones (a) - (f) -, la unidad del poema no aparece. Cabe, entonces, añadir otras nuevas observaciones:

(g) las equivalencias verbales: (gl) Hay-son-serản-(hay)-Hay; (g2) (hacen) abren, perteneciente a "hay golpes"; (hace) vuelve, perteneciente a "(hay) el hombre";

(h) los enunciados comparables precedidos por verbos (serán, son) -véase (d) - y por grupos adverbiales: (hl) "como del odio de Dios" (v. 2); (h2) "como si ante ellos, / la resaca de todo lo sufrido / se empozara en el alma" (vs. 2-4); (h3) "como / cuando por sobre el hombro nos llama una palmada" (vs. 13-14); (h4) "tal vez los potros de bárbaros atilas" (v. 7); (h5) "(tal vez) los heraldos negros que nos manda la Muerte" (v. 8);

(i) los enunciados precedidos por grupos verbales -cf. (g2) -: (i1) : " (hacen) abren zanjas oscuras" (v. 5) ; (i2) " (hace) vuelve los ojos locos" (v. 15) ;

(j) los enunciados exclamativos precedidos de proposiciones precedidas por Hay: (j1) ". . Yo no sé!" (v. 1) ; (j2) "...Pobre... pobre!" (v. 13); (j3) "...YYo no sé!" (v. 17).

La unidad del poema no aparece (aunque puedan hallarse todavía otros acoplamientos), ni puede aparecer en el agotamiento estático de la estructura. Para coordinar ésta de manera que las equivalencias encuentren su movimiento (y en consecuencia la unidad), es necesario "forzar" (ya volveré sobre este aspecto) el modelo de Levin, de la manera siguiente:

Entre golpes y hombre (vs. 1 y 13) se pueden establecer equivalencias T1, pero no es claro que puedan establecerse equivalencias T2. Por lo tanto, y de acuerdo con la fórmula (8), aquí no puede hablarse de acoplamiento: los dos enunciados son relacionales en el sintagma y, digamos, cuasi- relaciones en el paradigma. Ahora bien, hay en el poema un paso del condicional al presente (empozara $\rightarrow$ empoza, vs. 4, 16), paso que se opera en los vs. 7-9 (serán $\rightarrow$ son): a través de este paso es como se pueden ordenar las uniones que nos permitirán escribir desde otro 
punto de vista la fórmula (9), que allá aparecía a través de conceptos extraños al modelo.

Por lo tanto, retomando el paso del condicional al presente, se puede observar que la equivalencia posicional ( $\mathrm{T} 1$ ) entre golpes y hombre permite introducir en el modelo de Levin (de aqui el "forzarlo") una operación de transformación (que simbolizaré por $\Longrightarrow$ ). Con esta nueva noción puede establecerse que, a través del paso de los versos 7-9, se opera una transformación que hace de golpes el equivalente semántico de hombre (transformación sintáctica). Es decir, la transformación opera sobre la equivalencia $\mathrm{T} 2$, cosa que estructuralmente, y de acuerdo con el modelo de Levin, no sería posible establecer. De este modo, escribiendo (x) por "golpes" y (z) por "hombre", la fórmula (8) puede reescribirse:

$$
\mathrm{Cp}=\{\operatorname{Pg} \mathrm{T} 1(\mathrm{x}, \mathrm{z})\} \Longrightarrow\left\{\operatorname{Pg} \mathrm{T} 2\left(\mathrm{x}^{\prime}, \mathrm{z}^{\prime}\right)\right\}
$$

la cual puede leerse así: sobre la equivalencia $\mathrm{Tl}$ se opera una transformación que la hace convergente con $\mathrm{T} 2$.

Ahora bien, el haber entrado en el juego de Levin, y el haber introducido el concepto de transformación para explicar la unidad del poema, hace aparecer más problemas que soluciones. Señalaré algunos:

1) Establecer, también, acoplamientos por transformación, nos permite observar en el poema aspectos que no hubieran aparecido de otra manera. Así, por ejemplo, entre el v. 1 y el 17 se opera una transformación que va del primero al último a través de los quince versos intermedios, de tal modo que el v. 17 no es una repetición del v. 1 , sino una modificación suya. De la misma manera, la convergencia entre "Los Heraldos Negros" (título) y su reaparación en el v. 8 puede analizarse también sobre la base de la transformación. En el caso del título, "Los Heraldos Negros" está precedido sólo por el blanco de la página y en un contexto que podemos marcar 0 (cero) $-(0) \ldots$ / Los Heraldos Negros / ... (0) -, en tanto que en el v. 8 aparece en un contexto modificador -o (serán) / los heraldos negros que nos manda la Muerte-, lo cual abre el camino al encuentro de nuevas convergencias entre los enunciados que definen a "golpes", y en los cuales se encuentran "heraldos negros"/"muerte"/"Destino" /"odio de Dios"/"Cristos del alma".

2) Dejando de lado las transformaciones y volviendo a los acoplamientos, según los formula Levin, quedan otros aspectos por considerar. Si Levin, en otro trabajo ${ }^{10}$, concede especial atención a la grafía en poesia, ¿por qué, en su modelo, establece como predicado de las equivalencias T2 sólo la fonía y no la grafía? En general, se puede responder a esta pregunta afirmando que, como en el caso de Jakobson, se privilegia un aspecto de la poesía: el aspecto fónico (que corresponde con el desarrollo de un modelo lingüístico: el modelo fonológico) ${ }^{11}$. En mi

10 S. LEvin, "Internal and external deviation in poetry", Word, 21 (1965), 225-237.

11 La lingüística inglesa ha insistido sistemáticamente, más quizá que ninguna otra, en el paralelismo de la fonía y la grafía, poniendo en tela de juicio la tradición aristotélica según la cual sólo el lenguaje oral (fonía) "representa la experiencia 
ejemplo, es claro que los rasgos gráficos destacan Destino y Muerte en los vs. 8 y 10 . Y, en cuanto ellos son componentes de dos enunciados que definen golpes y metonimizan 'sufrir', se pueden establecer acoplamientos (introduciendo aquí de nuevo el concepto de transformación) convergentes con "Cristos del alma" (v. 9) y "odio de Dios" (v. 2), lo cual se enlaza con mis observaciones sobre el título y desemboca en la cuestión de los enunciados que llevan palabras en mayúscula.

3) Hay todavía un problema fundamental suscitado por el intento de dar cuenta de la unidad de un poema, y que puede formularse con las palabras de Jakobson cuando, refiriéndose a las coordenadas diacrónicas y sincrónicas en poesía, afirma: "La elección de una nueva corriente, dentro del caudal de los clásicos, o sea la reinterpretación que el poeta hace, establece los problemas esenciales para los estudios literarios sincrónicos"'12.

Con ayuda de algunos estudios generales sobre "César Vallejo"13 se pueden señalar, a título de simple información etnográfica, hechos como los siguientes:

a) Los Heraldos Negros es título no sólo del primer poema del libro, sino de todos los demás en él incluidos.

b) Además, "Los Heraldos Negros" (poema) es el primero de un sub-grupo de poemas delimitados por el título Plafones ágiles, lo cual nos remite a textos modernistas, no sólo por el primer poema del subgrupo ("Deshojación sagrada"), sino, fundamentalmente, por el galicismo plafones y su modificador ágiles. Incluso, "Deshojación sagrada" está centrado sobre la luna como figura poética, que constituye una verdadera obsesión entre los modernistas ${ }^{14}$.

c) Por otra parte, no puede verse como una casualidad el hecho de que el primer poema de Plafones ágiles tenga por título "Deshojación sagrada" y que haya un poema de Herrera y Reissig (anterior, claro está, a Los Heraldos Negros) intitulado "Desolación absurda": desolación y deshojación son, fónica y gráficamente, palabras muy similares, y $a b$ surda y sagrada son trisílabos graves, con acento prosódico en $2^{\mathrm{a}}$ sílaba.

d) En "Nochebuena", poema de Plafones ágiles, leemos estos versos:

Al callar la orquesta, pasean veladas sombras femeninas bajo los ramajes [...].

Hay labios que lloran arias olvidadas,

mental". Véase A. McINrosh, "Graphology and meaning", en Patterns of language, ed. McIntosh \& Halliday, London, 1966, pp. 98-110 (el trabajo es de 1961). Esta posición no ha sido aceptada por todos, y sigue manteniéndose aún el privilegio de la fonía sobre la grafía. Véase el trabajo de E. Alarcos Llorach recogido en Le langage, ed. A. Martinet, Paris, 1968. Las consecuencias filosófico-ideológicas de esta tradición han sido puestas de relieve por J. Derrida, De la grammatologie, Paris, 1967.

12 R. Jakobson, "Linguistics and poetics", art. cit., p. 352.

13 Entre otros, L. Monguió, César Vallejo: vida y obra, New York, 1952; A. Coyné, César Vallejo y su obra poética, Lima, 1957; G. Meo Zilio, Stile e poesia in César Vallejo, Padova, 1960. También RevIb, 36 (1970), núm. 71 (dedicado todo a Vallejo) .

14 Leopoldo Lugones le consagra todo un libro; Herrera y Reissig emplea ocho veces la figura en sólo quince páginas (Poesias completas, Buenos Aires, 1942, pp. 95110); el pre-modernista José Asunción Silva la emplea también muy a menudo. 
grandes lirios fingen los ebúrneos trajes $[\ldots]$

perfuman de seda los rudos boscajes [...],

en los cuales no puede dejar de verse la presencia de "Era un aire suave"15:

Es noche de fiesta, y el baile de trajes

ostenta su gloria de triunfos mundanos.

La divina Eulalia, vestida de encajes [...]

y el ebúrneo cisne sobre el quieto estanque $[\ldots]$

la marquesa alegre llegará al boscaje [...],

como también de "Luz de luna"16:

Anoche una fiesta con su grato bullicio

animaba de ese amor el tranquilo escenario [...]

¡Oh girar de desnudas espaldas!

$\mathrm{Oh}$ cadencias del valse que mueve

torbellinos de tules y gasas!

A base de los ejemplos aducidos, puede sacarse una primera conclusión. Un texto no es una unidad cerrada -aunque se proponga como tal (véase adelante) -, sino un diálogo permanente con otros textos. Este diálogo hace difícil formular el problema de la unidad, dado que todo texto -tomado para la lectura o para el análisis (la diferencia es aquí de grados, no de niveles) - se abre hacia la infinitud de textos existentes. La presencia del texto en el texto ha sido largamente estudiada, en la historia de la teoría y de la crítica literarias, con el nombre de "influencia". La noción de influencia tiene dos dificultades principales: privilegia al "influenciante" otorgando un papel pasivo al "influenciado", y hace aparecer el texto como "propiedad" de un "autor"17. El concebir el texto como diálogo con otros textos pone de manifiesto un aspecto oscurecido por la noción de influencia: el texto como producción. Para referirme a este entrecruzamiento de textos que acabo de señalar, me ha parecido necesario sustituir la noción de influencia por la de enunciados intertextuales ${ }^{\mathbf{1 8}}$.

En este diálogo con los textos modernistas, y con un deseo de subvertirlos, Los Heraldos Negros reescriben la textura romántica. En Silva, situado en el límite entre romanticismo y modernismo, los textos escriben todavía el amor-sacrificio; en Darío, escriben el amor-juego. En Los Heraldos Negros, el resultado de un trabajo de subversión del amorjuego conduce, paradójicamente, a la recuperación, por un lado, de la textura bíblica (ejemplos: "El poeta a su amada", y el título mismo del libro, que mediante el empleo de la sinestesia, figura cara a los moder-

15 Rubén Dario, Poesia, México, 1952, pp. 182-184.

16 José Asunción Silva, Poesias completas, Buenos Aires, s.a., pp. 109-111.

17 Sobre la influencia y el intertexto véase J. Aguilar Mora y W. Mignolo, "Borges, el libro y la escritura", Carav, 1971, núm. 17, 187-194.

$18 \mathrm{La}$ noción del intertexto, introducida por M. Bakhtine, es desarrollada por J. Kristeva en "Le mot, le dialogue et le roman", recogido en Recherches pour une manalyse, Paris, 1969, pp. 143-173. Como enunciado intertextual es analizado por W. Mignolo, Modèles et poétique, tesis inédita, Paris, 1972. 
nistas, guarda la armadura y transforma el mensaje), y, por otro lado, a reescribir los textos románticos, no sólo a través de palabras (Destino, Muerte, Duda, Misterio, etc.), sino fundamentalmente en la marca que tales enunciados producen (fatalidad, resignación). Y cabe señalar aún, como recuperación de textura romántica, las frases tomadas de la lengua cotidiana (cf. vs. 11-12 y 14) ${ }^{19}$.

El diálogo con los textos modernistas, escrito (producido) en una textura romántica y bíblica, constante a lo largo del libro, es reforzado por "Espergesia" (poema final), que claramente, tanto por su título ${ }^{20}$ como por su texto mismo, cierra el libro21. Esta constante, que opera a lo largo del libro, puede verse mejor a través de una rápida lectura de "La cena miserable". Miserable, como modificador de cena, opera en la misma forma que negros en relación con heraldos. Cena, además, en su función lingüística contextual ${ }^{22}$ y en las marcas que establece el poema, remite a sus connotaciones bíblicas, mientras que, por las mismas razones, miserable remite a textos románticos. Por otra parte, un análisis del poema mostraría cómo los pasivos se textualizan en relación con hombre, en tanto que los activos se textualizan en relación con un "más allá" del hombre (Dios, Destino). Dios, que aparece en todo el libro bajo un aspecto que podemos llamar "negativo" ("odio de Dios", "yo nací un día en que Dios estaba enfermo", "que como Dios engendra sin sensual placer", etc.), no se subvierte como noción (texto, escritura), sino que intensifica lo que ya estaba implícito: el castigo. Si la palabra (los enunciados) es (son) el lugar dialogante de todo texto ${ }^{23}$, Los Heraldos Negros, atravesado por poemas como "La cena miserable" y cerrado por "Espergesia", escribe lo que no siempre está escrito, pero que está siempre dicho. En el esquema que sigue,

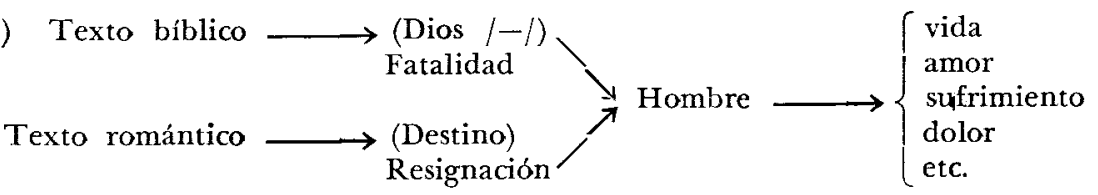

puede leerse cómo el acento, colocado sobre el aspecto negativo de Dios ("Espergesia", "Dios", "Amor"), reencuentra la resignación del Destino romántico ("Los Heraldos Negros") para insertar, en este "más allá" establecido con anterioridad a la escritura que los crea, todos los "temas"

19 Es conocida la importancia que los románticos dieron a la lengua coloquial. Recuérdese cómo Wordsworth decía que la lengua de los poetas se queda corta en comparación con lo que expresan los hombres, en la vida real, bajo la presión de las pasiones.

20 La espergesia es una figura de retórica que consiste en decir siempre la misma cosa, de maneras diferentes.

21 Julra Kristeva, "Le texte clos", Langages, 1968, núm. 12, 103-125, ha Ilamado la atención sobre el texto novelesco como texto cerrado; este hecho se corresponde con lo que ella llama idéologème du signe. Señalaré aquí, rápidamente, que la particularidad del texto cerrado no se refiere sólo a un género (novela), sino a todo texto que funda y mantiene ese idéologème du signe.

22 Cf. Tatiana Slama-Cazacu, Langage et contexte, La Haye, 1961.

23 Cf. M. Bakhtine, Problèmes de la poétique de Dostö̈evski, Paris, 1969. 
cuyo centro es el hombre y que a partir de él se generan (vida, amor, etcétera). Dicho más bruscamente: estos textos producen la inscripción del antropocentrismo en el teocentrismo.

Estas últimas consideraciones me han llevado mucho más lejos de lo que el modelo de Levin permite. No obstante, importa señalar que el esquema (11) está ya contenido en "Los Heraldos Negros" (cf. supra, pp. 404-407). Pero el modelo lingüístico no permite explicarlo. De ahí que mis observaciones estuvieran destinadas a señalar cómo los acoplamientos (que contienen el intertexto) se disuelven en los enunciados intertextuales. De ahí que sea una quimera ideológicamente discutible el mantener el a priori neoclásico de la unidad del poema. Estoy ya en condiciones de concluir y precisar lo que quiero decir cuando hablo de los "límites de la lingüística".

En primer lugar, fuerza es reconocer cuánto debe la poética, en vías de elaborarse en nuestros tiempos, al cuadro teórico abierto por la lingüística. La lingüística ha permitido que también para la poética sea válida la observación de $\mathrm{Hjemslev}^{24}$ : si una cierta tradición, en nombre del humanismo, niega la posibilidad de crear un lenguaje formalizado para dar cuenta de los hechos creados por el hombre (recuérdese a Leo Spitzer), entonces es preciso re-definir la noción de humanismo. Téngase en cuenta, además, que la noción de humanismo, creada a partir del Renacimiento, se inscribe en la producción de la "ideología del sujeto" que, con Descartes, pondrá al hombre en el centro del mundo. Si en ciertos aspectos la lingüística es aún deudora de tal herencia, es innegable que también abre el camino para su superación ${ }^{25}$.

En segundo lugar, hay que mencionar un problema que podemos llamar técnico: el paso de la hipótesis a la elaboración de un modelo ${ }^{26}$. La elaboración de modelos es un paso adelante en el despegue de las ataduras con los datos empíricos. El modelo tiene la ventaja de marcar, en sus mismos límites, la necesidad de corrección (de ahí la introducción del concepto de transformación, sugerida antes) ${ }^{27}$, y de permitir la elaboración de un discurso lógico que supere las tentativas de "descripción lingüística de textos literarios" consistentes en emplear el arsenal lingüístico según las necesidades de un poema dado ${ }^{28}$.

En tercer lugar está una fundamental cuestión que, una vęz más,

24 L. HJemslev, Prolégomènes à une théorie du langage, Paris, 1966, en especial pp. 17-21. ("Humanisme et théorie du langage").

25 Pienso en "los dos Saussure" de que habla J. Derrida, op. cit. Aqui me limito a la lingüistica, pero no hay que olvidar que la ruptura epistemológica (término que L. Althusser ha tomado de G. Bachelard) no es una particularidad exclusiva de ella.

26 Sobre la relación hipótesis-modelo en la lógica de la ciencia, véase R. Harré, An introduction to the logic of the sciences, London, 1960.

27 Críticas del modelo de Lcvin: N. RuweT, "L'analyse structurale en poésie", Ling, 1963, núm. 2, 38-59, y W. O. Hendricks, "Levin's linguistic structures in poetry", Lan, 42 (1966) , 639-648.

28 Por ejemplo, J. McH. SincLAIR, "Taking a poem to pieces", en Essays on style and language, ed. R. Fowler, London, 1966, pp. 68-81; G. N. LEECH, A linguistic guide to English poetry, London, 1969; N. Ruwer, "Je te donne ces vers...", Poétique, 1971, núm. 7, 388-401. Merece especial atención, por sus sugerencias para un modelo poéticolingüistico, el trabajo de M. A. K. HALLIDAY, "The linguistic study of literary texts", en Patterns of language, op. cit., pp. 56-69. 
puede resumirse en palabras de Jakobson: "Siendo la lingüística la ciencia de las estructuras lingüísticas, la poética puede considerarse como parte integrante de la lingüística" (art. cit., p. 351). Esta dependencia se mantiene aún, aunque no explícitamente, en los intentos de transposición de la lingüística transformacional para fundar una poética ${ }^{29}$. $Y$ aquí el problema es el siguiente: en esta actitud (imperialista), la lingüística pretendería ser el único discurso con derecho a agotar un dominio, y no simplemente a delimitar un objeto ${ }^{30}$. Si la lingüística intenta delimitar su objeto a partir del a priori de la diferencia entre lengua comunicacional y lengua poética, habrá que contestar que no es éste el único a priori capaz de delimitar un objeto. Tal como lo he señalado, especialmente al evocar los textos de Silva, Dario y Herrera y Reissig, es posible (dentro de las ciencias del lenguaje) elaborar modelos partiendo de la concepción del texto como práctica productiva -práctica productiva y translingüística que abre el camino a la elaboración de modelos semióticos ${ }^{31}$. Todo ello plantea una cuestión fundamental: la dispersión de la poética. En la medida en que el dominio es inagotable, el conocimiento se parcializa en la delimitación de diversos objetos (poético-lingüístico, poético-semiótico, poético-retórico, poético-estilístico) ${ }^{32}$. En cada una de estas tentativas, el problema, más epistemológico-ideológico que técnico, se puede formular así: en qué medida es posible elaborar un discurso lógico-teórico ( $y$, en consecuencia, delimitar un objeto) que nos conduzca a re-definir el humanismo en un marco ideológico que escape a la ideología de la conciencia y sea una subversión de esa ideología.

Walter Mignolo

Indiana University.

29 Cf. T. A. VAN DiJK, "Aspects d'une théorie générative du texte poétique", en Essais de sémiotique poétique, ed. J. Greimas, Paris, 1972, pp. 180-206.

30 objeto es el centro teórico que determina un discurso; dominio es el campo general en que la teoría se apoya (o a partir del cual procede) para elaborar su objeto. Objeto corresponde a lo que los físicos llaman sistema, o a lo que L. Althusser, Leer el "Capital", México, 1969, llama "objeto de conocimiento" por contraste con el "objeto real" (dominio).

31 J. KRISTEva, Recherches pour une sémanalyse, op. cit.; I. I. Revzin, "Dalla linguistica strutturale alla semiotica", en I sistemi di segni e lo strutturalismo sovietico, introd, di Faccani-Eco, Milano, 1969, pp. 55-72.

32 Idea desarrollada en W. Mignolo, Modèles et poétique, op. cit. Cuando digo "modelos poético-retóricos" o "poético-estilísticos" me refiero, obviamente, al replanteo a que estas disciplinas están sometidas en nuestros días (Riffaterre, Thorne, Dubois, Booth, etc.). 